\title{
HESS upper limits on very high energy gamma-ray emission from the microquasar GRS 1915+105
}

HESS Collaboration, F. Acero ${ }^{15}$, F. Aharonian ${ }^{1,13}$, A. G. Akhperjanian ${ }^{2}$, G. Anton ${ }^{16}$, U. Barres de Almeida ${ }^{8, \star}$, A. R. Bazer-Bachi ${ }^{3}$, Y. Becherini ${ }^{12}$, B. Behera ${ }^{14}$, K. Bernlöhr' ${ }^{1,5}$, A. Bochow ${ }^{1}$, C. Boisson ${ }^{6}$, J. Bolmont $^{19}$, V. Borrel $^{3}$, J. Brucker ${ }^{16}$, F. Brun ${ }^{19}$, P. Brun ${ }^{7}$, T. Bulik ${ }^{29}$, I. Büsching ${ }^{9}$, T. Boutelier ${ }^{17}$, P. M. Chadwick ${ }^{8}$, A. Charbonnier ${ }^{19}$, R. C. G. Chaves ${ }^{1}$, A. Cheesebrough ${ }^{8}$, J. Conrad ${ }^{31}$, L.-M. Chounet ${ }^{10}$, A. C. Clapson ${ }^{1}$, G. Coignet ${ }^{11}$, M. Dalton ${ }^{5}$, M. K. Daniel ${ }^{8}$, I. D. Davids ${ }^{22,9}$, B. Degrange ${ }^{10}$, C. Deil ${ }^{1}$, H. J. Dickinson ${ }^{8}$, A. Djannati-Atai ${ }^{12}$, W. Domainko ${ }^{1}$, L.O'C. Drury ${ }^{13}$, F. Dubois ${ }^{11}$, G. Dubus ${ }^{17}$, J. Dyks ${ }^{24}$, M. Dyrda ${ }^{28}$, K. Egberts ${ }^{1,30}$, P. Eger $^{16}$, P. Espigat ${ }^{12}$, L. Fallon ${ }^{13}$, C. Farnier ${ }^{15}$, S. Fegan ${ }^{10}$, F. Feinstein ${ }^{15}$, A. Fiasson ${ }^{11}$, A. Förster ${ }^{1}$, G. Fontaine ${ }^{10}$, M. Füßling ${ }^{5}$, S. Gabici ${ }^{13}$, Y. A. Gallant ${ }^{15}$, L. Gérard ${ }^{12}$, D. Gerbig ${ }^{21}$, B. Giebels ${ }^{10}$, J. F. Glicenstein ${ }^{7}$, B. Glück ${ }^{16}$, P. Goret ${ }^{7}$, D. Göring ${ }^{16}$, M. Hauser ${ }^{14}$, S. Heinz ${ }^{16}$, G. Heinzelmann ${ }^{4}$, G. Henri ${ }^{17}$, G. Hermann ${ }^{1}$, J. A. Hinton ${ }^{25}$, A. Hoffmann ${ }^{18}$, W. Hofmann ${ }^{1}$, P. Hofverberg ${ }^{1}$, M. Holleran ${ }^{9}$, S. Hoppe ${ }^{1}$, D. Horns ${ }^{4}$, A. Jacholkowska ${ }^{19}$, O. C. de Jager ${ }^{9}$, C. Jahn ${ }^{16}$, I. Jung ${ }^{16}$, K. Katarzyński ${ }^{27}$, U. Katz ${ }^{16}$, S. Kaufmann ${ }^{14}$, M. Kerschhaggl ${ }^{5}$, D. Khangulyan ${ }^{1}$, B. Khélifi ${ }^{10}$, D. Keogh ${ }^{8}$,

D. Klochkov ${ }^{18}$, W. Kluźniak ${ }^{24}$, T. Kneiske ${ }^{4}$, Nu. Komin $^{7}$, K. Kosack ${ }^{7}$, R. Kossakowski ${ }^{11}$, G. Lamanna ${ }^{11}$, J.-P. Lenain ${ }^{6}$, T. Lohse ${ }^{5}$, V. Marandon ${ }^{12}$, A. Marcowith ${ }^{15}$, J. Masbou ${ }^{11}$, D. Maurin ${ }^{19}$, T. J. L. McComb ${ }^{8}$, M. C. Medina ${ }^{6}$, J. Méhault ${ }^{15}$, R. Moderski ${ }^{24}$, E. Moulin ${ }^{7}$, M. Naumann-Godo ${ }^{10}$, M. de Naurois ${ }^{19}$, D. Nedbal ${ }^{20}$, D. Nekrassovi ${ }^{1}$, B. Nicholas ${ }^{26}$, J. Niemiec ${ }^{28}$, S. J. Nolan ${ }^{8}$, S. Ohm ${ }^{1}$, J-F. Olive ${ }^{3}$, E. de Oña Wilhelmi ${ }^{1}$, K. J. Orford ${ }^{8}$, M. Ostrowski ${ }^{23}$, M. Panter ${ }^{1}$, M. Paz Arribas ${ }^{5}$, G. Pedaletti ${ }^{14}$, G. Pelletier ${ }^{17}$, P.-O. Petrucci ${ }^{17}$, S. Pital ${ }^{12}$, G. Pühlhofer ${ }^{18}$, M. Punch ${ }^{12}$, A. Quirrenbach ${ }^{14}$, B. C. Raubenheimer ${ }^{9}$, M. Raue ${ }^{1,33}$, S. M. Rayner ${ }^{8}$, O. Reimer $^{30}$, M. Renaud $^{12}$, R. de los Reyes ${ }^{1}$, F. Rieger ${ }^{1,33}$, J. Ripken ${ }^{31}$, L. Rob ${ }^{20}$, S. Rosier-Lees ${ }^{11}$, G. Rowell ${ }^{26}$, B. Rudak ${ }^{24}$, C. B. Rulten ${ }^{8}$, J. Ruppel ${ }^{21}$, F. Ryde ${ }^{32}$, V. Sahakian ${ }^{2}$, A. Santangelo ${ }^{18}$, R. Schlickeiser ${ }^{21}$, F. M. Schöck ${ }^{16}$, A. Schönwald ${ }^{5}$, U. Schwanke ${ }^{5}$, S. Schwarzburg ${ }^{18}$, S. Schwemmer ${ }^{14}$, A. Shalchi ${ }^{21}$, I. Sushch ${ }^{5}$, M. Sikora ${ }^{24}$, J. L. Skilton ${ }^{25}$, H. Sol ${ }^{6}$, Ł. Stawarz $^{23}$, R. Steenkamp ${ }^{22}$, C. Stegmann ${ }^{16}$, F. Stinzing ${ }^{16}$, G. Superina ${ }^{10}$, A. Szostek ${ }^{23,17}$, P. H. Tam ${ }^{14}$, J.-P. Tavernet ${ }^{19}$, R. Terrier ${ }^{12}$, O. Tibolla ${ }^{1}$, M. Tluczykont ${ }^{4}$, C. van Eldik ${ }^{1}$, G. Vasileiadis ${ }^{15}$, C. Venter ${ }^{9}$, L. Venter ${ }^{6}$, J. P. Vialle ${ }^{11}$, P. Vincent ${ }^{19}$, M. Vivier ${ }^{7}$, H. J. Völk ${ }^{1}$, F. Volpe ${ }^{1}$, S. Vorobiov ${ }^{15}$, S. J. Wagner ${ }^{14}$, M. Ward ${ }^{8}$, A. A. Zdziarski ${ }^{24}$, and A. Zech ${ }^{6}$

(Affiliations can be found after the references)

Received 1 October 2009 / Accepted 21 October 2009

\section{ABSTRACT}

Context. High energy particles reside in the relativistic jets of microquasars, making them possible sources of very high energy radiation (VHE, $>100 \mathrm{GeV})$. Detecting this emission would provide a new handle on jet physics.

Aims. Observations of the microquasar GRS 1915+105 with the HESS telescope array were undertaken in 2004-2008 to search for VHE emission. Methods. Stereoscopic imaging of Cherenkov radiation from extensive air showers is used to reconstruct the energy and direction of the incident gamma rays.

Results. There is no evidence for a VHE gamma-ray signal either from the direction of the microquasar or its vicinity. An upper limit of $6.1 \times$ $10^{-13} \mathrm{ph} \mathrm{cm}^{-2} \mathrm{~s}^{-1}\left(99.9 \%\right.$ confidence level) is set on the photon flux above $410 \mathrm{GeV}$, equivalent to a VHE luminosity of $\sim 10^{34} \mathrm{erg} \mathrm{s}{ }^{-1}$ at $11 \mathrm{kpc}^{\text {. }}$ Conclusions. The VHE to X-ray luminosity ratio in GRS $1915+105$ is at least four orders of magnitude lower than the ratio observed in gamma-ray binaries. The VHE radiative efficiency of the compact jet is less than $0.01 \%$ based on its estimated total power of $10^{38} \mathrm{erg} \mathrm{s}^{-1}$. Particle acceleration in GRS $1915+105$ is not efficient at high energies and/or the magnetic field is too strong. It is also possible that VHE gamma-rays are produced by GRS 1915+105, but the emission is highly time-dependent.

Key words. X-rays: binaries - X-rays: individuals: GRS 1915+105 - gamma rays: observations

\section{Introduction}

Microquasars are X-ray binaries with relativistic jets. The jets are detected and resolved through their non-thermal radio emission. The well studied example is GRS $1915+105$, a system that has been known since the onset of its activity in 1992 (Castro-Tirado et al. 1992) and which has remained active until now. Identification of the companion star, followed by radial

\footnotetext{
* Supported by CAPES Foundation, Ministry of Education of Brazil.
}

velocity studies, showed that GRS $1915+105$ hosts a $\mathrm{K}$ giant with a mass $\leq 1.3 M_{\odot}$ and radius $20 R_{\odot}$ on a 33 day orbit around $\mathrm{a} \approx 14 M_{\odot}$ black hole (Harlaftis \& Greiner 2004). Soon after the discovery, apparent superluminal motions in two-sided radio knots moving away from the core with true velocity $\geq 0.9 c$ were reported by Mirabel \& Rodriguez (1994). The estimated distance to the microquasar is 8-13 kpc (Zdziarski et al. 2005).

Multi-wavelength observations from radio wavelengths to $\mathrm{X}$-rays have shown a very complex behavior. The X-ray 
variability of GRS $1915+105$ was interpreted to be transitions between three basic states, where some of the spectral changes could be related to a rapid disappearance of the inner portions of the accretion disc when material is ejected and the subsequent slower refilling of the emptied region (Belloni et al. 1997, 2000; Mirabel et al. 1998). A rich phenomenology is also observed in the radio domain. It includes radio quiet states, prolonged phases of relatively bright emission from a compact jet, strong radio flares associated with major ejections in the apparent superluminal jets and rapid radio oscillations (for a comprehensive review see Fender \& Belloni 2004; and Rushton et al. 2009). The X-ray and radio emission are known to be correlated with each other, which reflects a close coupling between the accretion disc and jet (Fender et al. 2004). In particular, there is a phenomenological relation between the X-ray "plateau" state and strong radio flares. The plateau state is characterized by a quasi-steady, optically-thick radio emission at the 50-200 mJy level, while the X-ray spectrum is dominated by a hard power-law component (Foster et al. 1996; Fender et al. 1999). It is sometimes related to one of the canonical states of X-ray binaries, namely the low/hard state (Klein-Wolt et al. 2002). This state is preceded and followed by a strong radio flare (Klein-Wolt et al. 2002). Moreover, just before the post-plateau flare, the X-ray spectrum softens for about 10-20 days (Fender et al. 1999; Klein-Wolt et al. 2002).

Superluminal jets in Galactic sources are places where particles can be accelerated to very high energies (VHE). Strong observational evidence exists for VHE electrons in the jets of two microquasars, namely XTE J1550-564 and H1743-322. In both cases, ejected plasma clouds were detected at distances of 0.1-1 pc from the binary. They showed a power-law spectrum extending from radio to X-rays that was presumably produced by a single electron distribution reaching TeV energies (Corbel et al. 2002, 2005). The highly energetic particles are expected to generate a very faint VHE emission as a result of inverse Compton scattering of ambient radiation fields. Particles accelerated much closer to the binary, where the radiation fields are stronger, could produce a detectable VHE flux. If the particle acceleration similar to that in XTE J1550-564 and H1743-322 takes place in GRS $1915+105$, the binary could emit VHE gamma-rays up to a few days after a major ejection event traced by a strong radio flare (Atoyan \& Aharonian 1999). The power of the jets in GRS $1915+105$ is estimated to exceed $10^{38} \mathrm{erg} \mathrm{s}^{-1}$ (Fender et al. 1999). Only a small fraction of this jet power needs to be converted to VHE gamma-rays in order to produce a detectable signal.

While the known sequence between the plateau state and major radio flares is helpful in scheduling VHE observations, the occurrence of flaring remains difficult to predict. Tantalizingly, the MAGIC collaboration claimed detection of VHE emission from another microquasar, Cyg X-1, during a bright X-ray flare that occurred unexpectedly and lasted several days (Albert et al. 2007a). Three other Galactic binaries have secure VHE detections. LS 5039 (Aharonian et al. 2006b) and LS I +61 303 (Albert et al. 2007b) contain an unidentified compact object in an orbit around an early-type star. In order to distinguish them from $\mathrm{X}$-ray binaries, due to the detected VHE emission and relatively low X-ray luminosities, LS 5039 and LS I +61 303 are classified as gamma-ray binaries. Both LS 5039 and LS I +61 303 display a steady orbital modulation of their VHE flux. However, the association of VHE emission with a relativistic jet is unclear. LS 5039 and LS I +61 303 could be rare examples of persistent, low X-ray luminosity microquasars (Romero et al. 2005; Paredes et al. 2006; Dermer \& Böttcher 2006). Alternatively, they could be rotation-powered (non-accreting) pulsars in interaction with their companion star (Dubus 2006) akin to the third gamma-ray binary PSR B1259-63 (Aharonian et al. 2005a).

Currently there is no evidence for gamma-ray or VHE gamma-ray emission from GRS $1915+105$. The binary is neither in the Third EGRET Catalog of High-Energy GammaRay Sources (Hartman et al. 1999) nor on a Fermi/Large Area Telescope bright gamma-ray source list (Abdo et al. 2009). The MAGIC telescope observed GRS $1915+105$ for $\sim 22 \mathrm{~h}$ and obtained an upper limit of $1.17 \times 10^{-12} \mathrm{ph} \mathrm{cm}^{-2} \mathrm{~s}^{-1}$ at the $95 \%$ confidence level, above $250 \mathrm{GeV}$ and assuming a power law spectrum $\left(\mathrm{d} N / \mathrm{d} E \sim E^{-\Gamma}\right)$ with the photon index $\Gamma=2.6$ (Saito et al. 2009). This corresponds to $0.7 \%$ of Crab Nebula flux.

The 1995-2000 Compton Gamma Ray Observatory satellite observations showed that the hard X-ray spectrum of GRS $1915+105$ is well fitted by a power law with $\Gamma=3$ and extending up to $1 \mathrm{MeV}$ without a cut off (Zdziarski et al. 2001). The extrapolation of this spectrum above $400 \mathrm{GeV}$ predicts a photon flux of only $10^{-16} \mathrm{ph} \mathrm{cm}^{-2} \mathrm{~s}^{-1}$, which is far below the MAGIC upper limit.

The other possible sources of VHE gamma-ray emission related to GRS $1915+105$ are the two small radio and infrared clouds positioned nearly symmetrically at an angular separation of $17^{\prime}$ from the system IRAS $19124+1106$ and IRAS $19132+1035$. Because their position angle is very similar to the position angle of the sub-arcsec radio jets from GRS 1915+105, they have been postulated to be associated with the termination of the jets in the interstellar medium (ISM) (Rodriguez \& Mirabel 1998; Chaty et al. 2001). But as discussed by Zdziarski et al. (2005) their association with GRS 1915+105 is highly uncertain.

HESS observations of GRS 1915+105 and both IRAS 19124+1106 and IRAS 19132+1035 are reported here and the findings are discussed in the context of the established variable multi-wavelength behavior.

\section{HESS data and analysis}

HESS is an array of four imaging atmospheric Cherenkov telescopes situated in the Khomas Highland of Namibia (Aharonian et al. 2006a). GRS 1915+105 was observed between 2004 and 2008 on several occasions, as a target of opportunity (ToO) or as a source in the field of view in other HESS pointings. After applying the standard HESS data quality selection criteria (Aharonian et al. 2006a), a total of $T_{\text {live }}=24.1 \mathrm{~h}$ live time were available for the analysis. The zenith angle of the observations varied between $33^{\circ}$ and $52^{\circ}$ with a mean value of $Z_{\text {mean }}=37^{\circ}$. GRS $1915+105$ was offset from the pointing direction of the telescope by $\psi$ in the range of $0.45^{\circ}-1.79^{\circ}$. Table 1 lists the dates of all observations which passed the quality checks and were included in the analysis. In accordance with the HESS guidelines, all the results presented below have been successfully cross-checked with an independent analysis and calibration chain (de Naurois 2006; de Naurois \& Rolland 2009).

A point source analysis was performed at the location of GRS $1915+105$ using the standard analysis techniques and selection cuts with an angular cut of $\theta^{2} \leq 0.0125 \mathrm{deg}^{2}$ and a size cut of 80 photo-electrons (Aharonian et al. 2004, 2005b). The resulting post-analysis energy threshold $E_{\text {th }}$ at $Z_{\text {mean }}$ is $410 \mathrm{GeV}$. The estimation of the background was done using the Reflected Background method for all the observation segments (Berge et al. 2007). A total number of source events $N_{\mathrm{ON}}=1052$ and background events $N_{\mathrm{OFF}}=15790$ (with a background to source 
Table 1. Observations of GRS 1915+105.

\begin{tabular}{lll}
\hline \hline Year & MJD-50000 & Subsets \\
\hline 2004 & $3123(4), 3124(4), 3125(3), 3126,3127(3), 3128$ & I, plateau \\
& $3141(4), 3142$ & I, post-flare \\
2005 & 3528 & \\
2006 & $3878,3879(2), 3887,3890(2), 3894$ & II \\
& $3964,3967(2), 3969(2), 3970,3974,3975$ & III \\
2008 & $4617(2), 4618(4), 4620(2), 4621(2), 4622(2), 4623(2), 4624(3), 4625(2), 4626$ & IV, plateau \\
\hline
\end{tabular}

${ }^{a}$ Figures in brackets indicate the number of independent observation segments ( $\sim 28$ min each) on a given night.

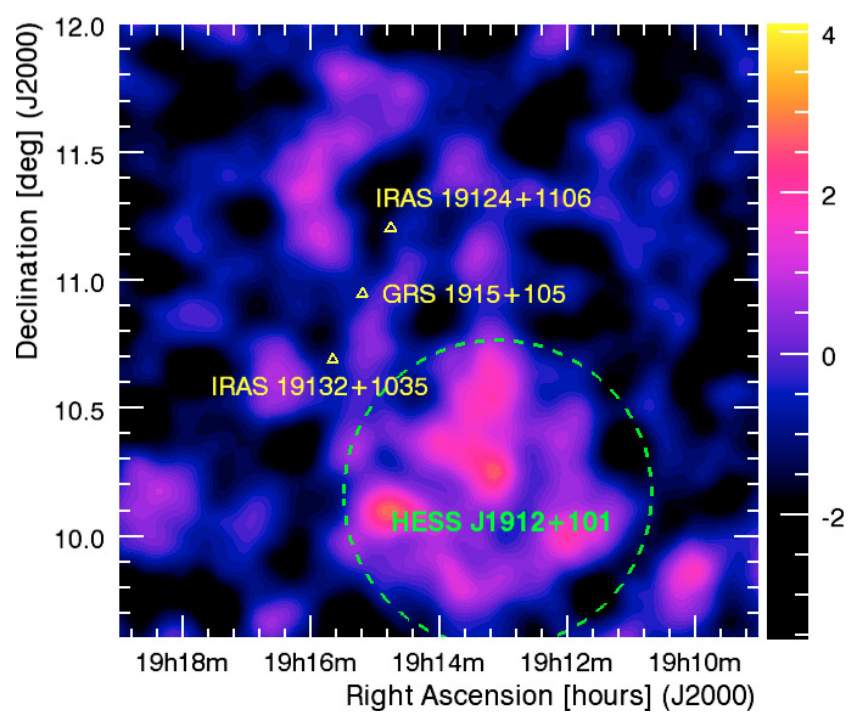

Fig. 1. Smoothed significance map of the region around GRS $1915+105$. The integration radius is $0.11^{\circ}$, and the map has been smoothed with a two-dimensional Gaussian of a radius of $0.09^{\circ}$. The triangles denote GRS $1915+105$ and the two IRAS sources, candidates for jet-ISM interaction sites. The background has been subtracted using the ring background method with the ring radius of $1^{\circ}$. The dotted circle corresponds to an exclusion region around HESS J1912+101. The data inside are not taken into account in the background calculations. Note that the map was not optimized for the presentation of the extended source HESS J1912+101, and its morphology may differ from that presented in the HESS discovery paper by Aharonian et al. (2008). In principle Aharonian et al. (2008) used an integration radius of $0.22^{\circ}$, which is twice as large, and smoothed the image with a two-dimensional Gaussian of a radius of $0.13^{\circ}$.

normalization $\alpha=0.067)$ were measured. This provides an excess of -5.9 events. The total significance $(S)$ of the excess from the direction of GRS 1915+105 (calculated using Eq. (17) of Li \& Ma 1983) is -0.3 standard deviations $(\sigma)$. Figure 1 shows a significance map of excess events around the position of GRS 1915+105 and Fig. 2 the distribution of the squared angular distance of observed gamma-ray candidates from the center of the binary in comparison to background data. The angular distribution of the source region events is compatible with the distribution of the background region events. There is no evidence for a VHE gamma-ray signal from GRS 1915+105.

The approach of Feldman \& Cousins (1998) is used to calculate the upper limits on the integrated photon flux above $410 \mathrm{GeV}$. The upper limit for the whole data set at the $99.9 \%$ confidence level is

$I(>410 \mathrm{GeV})<6.1 \times 10^{-13} \mathrm{ph} \mathrm{cm}^{-2} \mathrm{~s}^{-1}$,

assuming a power law with the photon index $\Gamma=2.5$. This corresponds to $0.7 \%$ of the Crab Nebula flux above the same energy

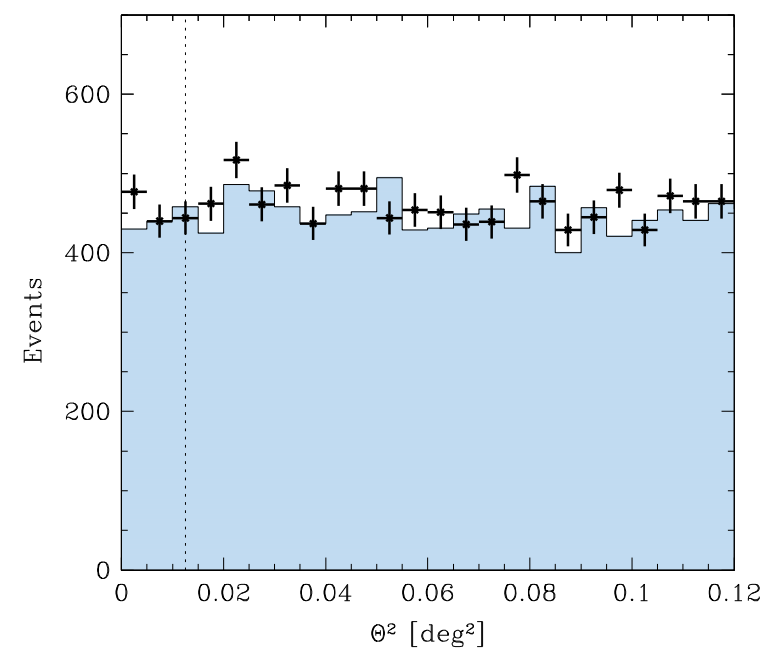

Fig. 2. Distribution of events as a function of the squared angular distance from GRS1915+105 for gamma-ray-like events in the ON-region (points) and from the centre of the OFF-regions (filled area). The vertical dotted line denotes the standard selection cut for point sources used by HESS.

threshold. The $95 \%$ confidence level for the same $\Gamma$ and energy threshold is

$I(>410 \mathrm{GeV})<3.2 \times 10^{-13} \mathrm{ph} \mathrm{cm}^{-2} \mathrm{~s}^{-1}$,

which corresponds to $0.4 \%$ of the Crab Nebula flux with the same energy threshold. The values depend only weakly on the assumed photon index (for photon indices from 2.0 to 3.5, the $99.9 \%$ upper limit changes from 0.8 to $0.6 \% \mathrm{Crab}$ ). The systematic error on the flux measurements is estimated to be $20 \%$ (Aharonian et al. 2006a). The upper limits are more stringent than the MAGIC results (Saito et al. 2009, Sect. 1).

The $R X T E^{1}$ All Sky Monitor (ASM) $1.5-12 \mathrm{keV}$ and Swift ${ }^{2}$ Burst Alert Telescope (BAT) 15-50 keV lightcurves are shown in Fig. 3 along with the dates when GRS $1915+105$ was observed by HESS. Because of the extreme source variability, the analysis was repeated separately for six data subsets. Four subsets (I, II, III, IV) have been chosen to correspond to well defined in time, quasi-continuous HESS observing periods - compare Table 1 and Fig. 3. Note that there is a single observation segment on 53528 MJD that is not considered as a separate subset.

The remaining two subsets contain data grouped by the X-ray spectral state. Two-thirds of the data (a part of subset I and the entire subset IV, $15 \mathrm{~h}$ livetime) correspond to the plateau state, a well defined state with uniform X-ray and radio properties. The last subset consists of five post-flare observational segments (all part of subset I), taken three days after a major

\footnotetext{
${ }^{1}$ Quick-look results provided by the RXTE/ASM team.

2 Swift/BAT transient monitor results provided by the Swift/BAT team.
} 
Table 2. The standard HESS analysis results for GRS 1915+105.

\begin{tabular}{|c|c|c|c|c|c|c|c|c|c|c|c|}
\hline Set & $\begin{array}{l}T_{\text {live }} \\
{[\mathrm{h}]}\end{array}$ & $\begin{array}{c}S \\
{[\sigma]}\end{array}$ & $N_{\mathrm{ON}}$ & $N_{\mathrm{OFF}}$ & $\alpha$ & $\begin{array}{c}Z_{\text {mean }} \\
{\left[{ }^{\circ}\right]}\end{array}$ & $\begin{array}{c}\psi_{\text {mean }} \\
{\left[{ }^{\circ}\right]}\end{array}$ & $\begin{array}{c}E_{\mathrm{th}} \\
{[\mathrm{GeV}]}\end{array}$ & $\begin{array}{c}I_{99.9 \%}\left(>E_{\mathrm{th}}\right) \\
{\left[10^{-12} \mathrm{ph} \mathrm{cm}^{-2} \mathrm{~s}^{-1}\right]}\end{array}$ & $\begin{array}{c}\text { Crab } \\
\%\end{array}$ & $\overline{P P\left(\chi^{2}\right)}$ \\
\hline All & 24.07 & -0.3 & 1052 & $\overline{15790}$ & 0.067 & 38 & 1.22 & 410 & $<0.61$ & $<0.7$ & 0.53 \\
\hline I & 8.56 & -0.8 & 508 & 6484 & 0.081 & 36 & 0.65 & 410 & $<0.84$ & $<1.0$ & 0.47 \\
\hline II & 2.86 & 0 & 96 & 2751 & 0.035 & 43 & 1.83 & 500 & $<2.22$ & $<3.4$ & 0.37 \\
\hline III & 3.52 & 0.3 & 102 & 1926 & 0.051 & 44 & 1.11 & 540 & $<1.90$ & $<3.3$ & 0.13 \\
\hline IV & 8.68 & 0.5 & 337 & 4204 & 0.078 & 36 & 0.70 & 400 & $<1.58$ & $<1.8$ & 0.62 \\
\hline Plateau & 15.54 & 0 & 746 & 9771 & 0.076 & 36 & 0.70 & 410 & $<0.98$ & $<1.1$ & 0.67 \\
\hline Post-flare & 1.70 & -0.9 & 99 & 917 & 0.118 & 35 & 0.50 & 400 & $<1.34$ & $<1.5$ & - \\
\hline
\end{tabular}
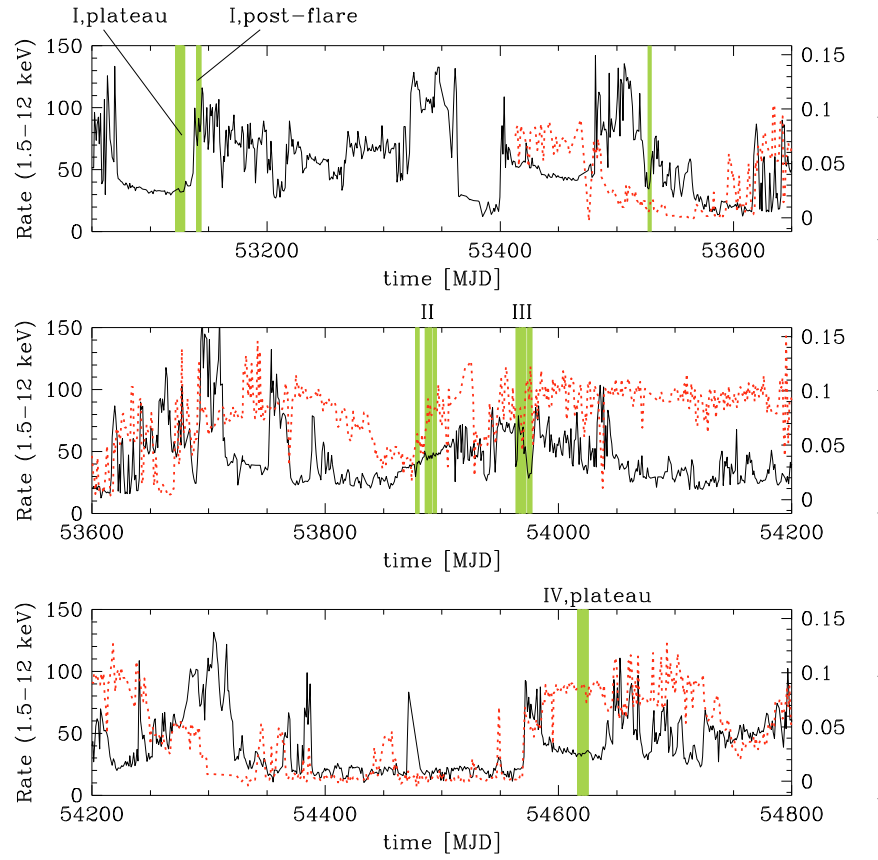

Fig. 3. GRS1915+105 1.5-12 keV RXTE/ASM lightcurve with lefthand scales in units of counts $\mathrm{s}^{-1}$ is drawn in black/solid lines; 15-50 keV Swift/BAT lightcurve with right-hand scales in units of cts $\mathrm{cm}^{-2} \mathrm{~s}^{-1}$ is drawn in red/dotted lines. The HESS observations are marked by green stripes, and the subset designations are placed above each panel.

radio flare. All subsets are defined in Table 1 and in Fig. 3. The results of the analysis are given in Table 2. There is no detectable gamma-ray signal in any of the subsets.

Observations from subsets I and IV were taken as a part of the ToO program aimed to observe GRS $1915+105$ during a major radio flare. Based on the relation between the plateau states and major radio ejections (Sect. 1), the observations were triggered during the plateau state defined as a radio flux of $\approx 60 \mathrm{mJy}$ level and a RXTE/ASM hardness ratio (5-12 keV/3-5 keV) above 1.7. Although in 2004 and 2008 GRS 1915+105 was observed for six and nine consecutive nights respectively, the source did not flare. In both cases the flare occurred days or weeks later respectively.

Lightcurves of nightly integral fluxes were examined to look for gamma-ray flares in each of the datasets. The resulting $\chi^{2}$ probability $P\left(\chi^{2}\right)$ for the fit to a constant flux, given in Table 2, shows no evidence for variability. The error of the nightly flux point equals $\sim 2 \%$ of Crab nebula (for $410 \mathrm{GeV}$ energy threshold) around a zero average nightly flux. A flare with a flux of $10 \%$ of the Crab $\left(8.6 \times 10^{-12} \mathrm{ph} \mathrm{cm}^{-2} \mathrm{~s}^{-1}\right)$ would have been detected in the $1 \mathrm{~h}$ of the HESS observation at the $5 \sigma$ level (pre-trial).
A point source analysis was also performed with the same parameter setup of the two infrared clouds, potential spots where the jets of GRS 1915+105 interact with ISM. Both sources are marked in Fig. 1. The significances and flux upper limits at the $99.9 \%$ confidence level above $410 \mathrm{GeV}$ for an assumed power law spectrum with photon index $\Gamma=2.5$ are for IRAS $19124+1106 S=1.1 \sigma$ and $I(>410 \mathrm{GeV})<$ $1.3 \times 10^{-12} \mathrm{ph} \mathrm{cm}^{-2} \mathrm{~s}^{-1}$ whereas for IRAS $19132+1035$ $S=0.1 \sigma$ and $I(>410 \mathrm{GeV})<4.8 \times 10^{-13} \mathrm{ph} \mathrm{cm}^{-2} \mathrm{~s}^{-1}$.

\section{Discussion and conclusions}

There is no evidence for a VHE gamma-ray signal from the direction of the microquasar and in its vicinity. An upper limit of $6.1 \times 10^{-13} \mathrm{ph} \mathrm{cm}^{-2} \mathrm{~s}^{-1}$ (99.9\% confidence level) is set on the photon flux above $410 \mathrm{GeV}$, equivalent to a VHE luminosity of $\sim 10^{34} \mathrm{erg} \mathrm{s}^{-1}$ at $11 \mathrm{kpc}$.

Radio observations of GRS 1915+105 sometimes show optically thin flares, corresponding to discrete relativistic ejection episodes, and sometimes show a steady optically thick emission associated with a compact jet. Both cases could be associated with VHE emission. Unlike for Cyg X-1 or LS 5039, VHE photon absorption due to pair creation on stellar photons is not expected to be an issue in GRS 1915+105: the opacity above $400 \mathrm{GeV}$ is $\lesssim 0.1$ assuming a $4500 \mathrm{~K}, 20 R_{\odot}$ stellar companion in a 33 day orbit around the black hole.

Atoyan \& Aharonian (1999) modeled the radio flares from GRS $1915+105$ as a synchrotron emission of expanding plasma clouds ejected from the system. The expected synchrotron selfCompton VHE emission depends strongly on the maximum energy to which particles are accelerated and on the value of the magnetic to kinetic energy in the ejecta. Equipartition implies a magnetic field strength of about $0.2 \mathrm{G}$ and very little VHE emission. Assuming an acceleration of electrons to $10 \mathrm{TeV}$ and a subequipartition magnetic field of $0.05 \mathrm{G}$, the VHE gamma-ray flux is expected to be comparable to that of the Crab nebula during the first hours of a strong radio outburst, declining to $\lesssim 10 \%$ of the Crab flux over a period of a few days. These fluxes would be detectable with the HESS telescopes. Although there are no HESS observations strictly simultaneous with a large radio flare, there are $1.7 \mathrm{~h}$ of observations taken three days after a moderately strong radio flare. The flux upper limit from this dataset is $1.5 \%$ of Crab (post-flare subset in Table 2). The non-detection suggests either a sub-TeV cut-off in the electron energy distribution and/or a magnetic field strength closer to the equipartition value. A detection in X-rays of the discrete ejecta would imply TeV electron energies, as in XTE J1550-564 and H1743-322 (Corbel et al. 2002, 2005). The HESS upper limits would then rule out sub-equipartition magnetic fields. However, the angular separation of the radio ejecta in GRS $1915+105$ is smaller $\left(\lesssim 1^{\prime \prime}\right)$ 
than in these other sources $\left(5^{\prime \prime}-20^{\prime \prime}\right)$, making the X-ray observation difficult.

VHE emission might also be expected from the steady, compact jet. A VHE flare in Cyg X-1 reached $4 \sigma$ in 153 min of observation time and had a luminosity of $\sim 10^{34} \mathrm{erg} \mathrm{s}^{-1}$ (Albert et al. 2007a). It occurred when the source was in the hard X-ray power-law state which is associated with bright, steady radio emission which is thought to arise in a conical, continuous, selfabsorbed compact jet (Blandford \& Königl 1979) with the total power $\sim 10^{36}-10^{37} \mathrm{erg} \mathrm{s}^{-1}$ (Gallo et al. 2005). During the Cyg X-1 VHE gamma-ray event, $\sim 0.1-1 \%$ of the total jet power was converted into VHE gamma-rays.

A compact jet with an estimated total power $\sim 3 \times 10^{38} \mathrm{erg} \mathrm{s}^{-1}$ (Fender \& Pooley 2000) is also present in GRS 1915+105 during the long plateau states (Klein-Wolt et al. 2002). If the jet power conversion efficiency were similar to that of Cyg X-1 during the VHE event, GRS $1915+105$ would be one of the brightest VHE gamma-ray sources in the sky. Meanwhile, for the HESS VHE gamma-ray flux upper limit the compact jet power conversion efficiency in GRS $1915+105$ is only $\sim 0.003 \%$. The large difference in jet conversion efficiencies between GRS 1915+105 and Cyg X-1 may be related to fact that the hard state of Cyg X-1 was exceptionally bright for a few days around the time of the VHE detection (Malzac et al. 2008), whereas the plateau states of GRS 1915+105 coincident with HESS observations did not have unusually high hard X-ray fluxes. In addition a distance difference between GRS 1915+105 at $11 \mathrm{kpc}$ and $\mathrm{Cyg} \mathrm{X}-1$ at $2.2 \mathrm{kpc}$ translates into a $\sim 25$ times lower flux at Earth, therefore events with a luminosity similar to that during the Cyg X-1VHE flare could not have been detected from GRS 1915+105.

Note also that Cyg X-1 is not a steady VHE source in its regular hard X-ray state, for which the MAGIC upper limit is $\sim 10^{33} \mathrm{erg} \mathrm{s}^{-1}$. The lack of persistent detectable VHE emission from the compact jets in Cyg X-1 and GRS 1915+105 is in agreement with the results of Bosch-Ramon et al. (2006), who studied the steady VHE gamma-ray emission expected from microquasar compact leptonic jets in the low/hard X-ray state. In the case of both the low-mass X-ray binaries (such as GRS 1915+105) and the high-mass X-ray binaries (such as Cyg X-1) the expected VHE luminosities are generally low at the level of $10^{32} \mathrm{erg} \mathrm{s}^{-1}$. The low $\mathrm{TeV}$ emission is also predicted for low mass microquasars with proton dominated jets where the VHE gamma-ray emission due to photoion production is either too weak or is suppressed by internal absorption (Romero \& Vila 2008).

The VHE to X-ray luminosity ratio in GRS $1915+105$ is also different from the case of the gamma-ray binaries LS 5039 and LS I +61 303, where typically the X-ray and VHE luminosities are similar. On the other hand, the radio fluxes are on the order of 10-100 mJy in gamma-ray binaries and GRS 1915+105. This applies to the plateau state of GRS $1915+105$, which has optically thick radio spectra, whilst the radio spectra of gammaray binaries suggest optically thin emission. Strict proportionality and analogy between GRS 1915+105 and gamma-ray binaries would have meant detection of a steady VHE flux from GRS 1915+105. The lack of detection suggest that if the gammaray binaries LS 5039 and LS I +61 303 are microquasars, then the channels through which their accretion energy is released are very different from those in GRS $1915+105$. It may also imply that no accretion or jet production takes place in gammaray binaries and the VHE photons are produced via pulsar and stellar wind interaction. In the latter case, any comparison with GRS $1915+105$ is unjustified.
There is no evidence for VHE gamma-ray emission from either of the two infrared clouds which could be related to the GRS 1915+105 jet termination shocks. Bordas et al. (2009) studied the details of the microquasar jet interaction with the ISM and found that the integrated fluxes above $100 \mathrm{GeV}$ from jets with power well above $10^{37} \mathrm{erg} \mathrm{s}^{-1}$ in a dense environments (particle density $>1 \mathrm{~cm}^{-3}$ ) might be detectable with the next generation of Cherenkov facilites like CTA, which aim for a 10 times improvement in sensitivity over current generation instruments.

Finally, it is also possible that the VHE emission of GRS $1915+105$ is highly time-dependent. It would be desirable to have observations simultaneously with one of the large, optically thin radio flares associated with major ejection events. There are known observable signs of impending major flares (Sect. 1), but these still require long, dedicated campaigns of tens of days to increase the chances of catching them. Despite present efforts, these remain difficult to organize with groundbased Cherenkov Telescope arrays. The all-sky monitoring abilities in the $0.1-100 \mathrm{GeV}$ range of the Fermi Gamma-ray Space Telescope are better suited to detecting transient emission. The typical flux that can be detected in hours is $\sim 10^{-6} \mathrm{ph} \mathrm{cm}^{-2} \mathrm{~s}^{-1}$ $(>100 \mathrm{MeV})$ or $\sim 10^{36} \mathrm{erg} \mathrm{s}^{-1}$ at $11 \mathrm{kpc}$. Fermi gamma-ray space telescope and future Cherenkov telescope arrays should help in understanding the time-dependent gamma-ray emission from microquasars.

Acknowledgements. The support of the Namibian authorities and of the University of Namibia in facilitating the construction and operation of HESS is gratefully acknowledged, as is the support by the German Ministry for Education and Research (BMBF), the Max Planck Society, the French Ministry for Research, the CNRS-IN2P3 and the Astroparticle Interdisciplinary Programme of the CNRS, the UK Science and Technology Facilities Council (STFC), the IPNP of the Charles University, the Polish Ministry of Science and Higher Education, the South African Department of Science and Technology and National Research Foundation, and by the University of Namibia. We appreciate the excellent work of the technical support staff in Berlin, Durham, Hamburg, Heidelberg, Palaiseau, Paris, Saclay, and in Namibia in the construction and operation of the equipment.

\section{References}

Abdo, A. A., Ackermann, M., Ajello, M., et al. 2009, ApJS, 183, 46

Aharonian, F., Akhperjanian, A. G., Aye, K.-M., et al. 2004, Astropart. Phys., 22, 109 (HESS Collaboration)

Aharonian, F., Akhperjanian, A. G., Aye, K.-M., et al. 2005a, A\&A, 442, 1 (HESS Collaboration)

Aharonian, F., Akhperjanian, A. G., Aye, K.-M., et al. 2005b, A\&A, 430, 865 (HESS Collaboration)

Aharonian, F., Akhperjanian, A. G., Bazer-Bachi, A. R., et al. 2006a, A\&A, 457, 899 (HESS Collaboration)

Aharonian, F., Akhperjanian, A. G., Bazer-Bachi, A. R., et al. 2006b, A\&A, 460, 743 (HESS collaboration)

Aharonian, F., Akhperjanian, A. G., Barres de Almeida, U., et al. 2008, A\&A, 484, 435 (HESS Collaboration)

Albert, J., Aliu, E., Anderhub, H., et al. 2007a, ApJ, 665, L51 (MAGIC Collaboration)

Albert, J., Aliu, E., Anderhub, H., et al. 2007b, ApJ, 665, L51 (MAGIC Collaboration)

Atoyan, A. M., \& Aharonian, F. A. 1999, MNRAS, 302, 253

Belloni, T., Mendez, M., King, A. R., van der Klis, M., \& van Paradijs, J. 1997, ApJ, 488, L109

Belloni, T., Klein-Wolt, M., Méndez, M., van der Klis, M., \& van Paradijs, J. 2000, A\&A, 355, 271

Berge, D., Funk, S., \& Hinton, J. 2007, A\&A, 466, 1219

Blandford, R. D., \& Königl, A. 1979, ApJ, 232, 34

Bordas, P., Bosch-Ramon, V., Paredes, J. M., \& Perucho, M. 2009, A\&A, 497, 325

Bosch-Ramon, V., Romero, G. E., \& Paredes, J. M. 2006, A\&A, 447, 263

Castro-Tirado, A. J., Brandt, S., \& Lund, N. 1992, IAU Circ., 5590, 2

Chaty, S., Rodríguez, L. F., Mirabel, I. F., et al. 2001, A\&A, 366, 1035

Corbel, S., Fender, R. P., Tzioumis, A. K., et al. 2002, Science, 298, 196

Corbel, S., Kaaret, P., Fender, R. P., et al. 2005, ApJ, 632, 504 
de Naurois, M. 2006 [arXiv: 0607247]

de Naurois, M., \& Rolland, L. 2009, Astropart. Phys., 32, 231

Dermer, C. D., \& Böttcher, M. 2006, ApJ, 643, 1081

Dubus, G. 2006, A\&A, 456, 801

Feldman, G. J., \& Cousins, R. D. 1998, Phys. Rev. D, 57, 3873

Fender, R., \& Belloni, T. 2004, ARA\&A, 42, 317

Fender, R. P., \& Pooley, G. G. 2000, MNRAS, 318, L1

Fender, R. P., Garrington, S. T., McKay, D. J., et al. 1999, MNRAS, 304, 865

Fender, R. P., Belloni, T. M., \& Gallo, E. 2004, MNRAS, 355, 1105

Foster, R. S., Waltman, E. B., Tavani, M., et al. 1996, ApJ, 467, L81

Gallo, E., Fender, R., Kaiser, C., et al. 2005, Nature, 436, 819

Harlaftis, E. T., \& Greiner, J. 2004, A\&A, 414, L13

Hartman, R. C., Bertsch, D. L., Bloom, S. D., et al. 1999, ApJS, 123, 79

Klein-Wolt, M., Fender, R. P., Pooley, G. G., et al. 2002, MNRAS, 331, 745

Li, T.-P., \& Ma, Y.-Q. 1983, ApJ, 272, 317

Malzac, J., Lubiński, P., Zdziarski, A. A., et al. 2008, A\&A, 492, 527

Mirabel, I. F., \& Rodriguez, L. F. 1994, Nature, 371, 46

Mirabel, I. F., Dhawan, V., Chaty, S., et al. 1998, A\&A, 330, L9

Paredes, J. M., Bosch-Ramon, V., \& Romero, G. E. 2006, A\&A, 451, 259

Rodriguez, L. F., \& Mirabel, I. F. 1998, A\&A, 340, L47

Romero, G. E., \& Vila, G. S. 2008, A\&A, 485, 623

Romero, G. E., Christiansen, H. R., \& Orellana, M. 2005, ApJ, 632, 1093

Rushton, A., Spencer, R. E., Pooley, G., \& Trushkin, S. 2009, MNRAS, submitted [arXiv: 0910.1779]

Saito, T. Y., Zanin, R., Bordas, P., et al. 2009 [arXiv : 0907. 1017]

Zdziarski, A. A., Grove, J. E., Poutanen, J., Rao, A. R., \& Vadawale, S. V. 2001, ApJ, 554, L45

Zdziarski, A. A., Gierliński, M., Rao, A. R., Vadawale, S. V., \& Mikołajewska, J. 2005, MNRAS, 360, 825

1 Max-Planck-Institut für Kernphysik, PO Box 103980, 69029 Heidelberg, Germany

2 Yerevan Physics Institute, 2 Alikhanian Brothers St., 375036 Yerevan, Armenia

3 Centre d'Étude Spatiale des Rayonnements, CNRS/UPS, 9 Av. du Colonel Roche, BP 4346, 31029 Toulouse Cedex 4, France

4 Universität Hamburg, Institut für Experimentalphysik, Luruper Chaussee 149, 22761 Hamburg, Germany

5 Institut für Physik, Humboldt-Universität zu Berlin, Newtonstr. 15, 12489 Berlin, Germany

6 LUTH, Observatoire de Paris, CNRS, Université Paris Diderot, 5 Place Jules Janssen, 92190 Meudon, France

7 IRFU/DSM/CEA, CE Saclay, 91191 Gif-sur-Yvette Cedex, France

8 University of Durham, Department of Physics, South Road, Durham DH1 3LE, UK

9 Unit for Space Physics, North-West University, Potchefstroom 2520, South Africa

10 Laboratoire Leprince-Ringuet, École Polytechnique, CNRS/IN2P3, 91128 Palaiseau, France

11 Laboratoire d'Annecy-le-Vieux de Physique des Particules, Université de Savoie, CNRS/IN2P3, 74941 Annecy-le-Vieux, France
12 Astroparticule et Cosmologie (APC), CNRS, Université Paris 7 Denis Diderot, 10 rue Alice Domon et Leonie Duquet, 75205 Paris Cedex 13; UMR 7164, CNRS, Université Paris VII, CEA, Observatoire de Paris, France

13 Dublin Institute for Advanced Studies, 5 Merrion Square, Dublin 2, Ireland

14 Landessternwarte, Universität Heidelberg, Königstuhl, 69117 Heidelberg, Germany

15 Laboratoire de Physique Théorique et Astroparticules, Université Montpellier 2, CNRS/IN2P3, CC 70, Place Eugène Bataillon, 34095 Montpellier Cedex 5, France

16 Universität Erlangen-Nürnberg, Physikalisches Institut, ErwinRommel-Str. 1, 91058 Erlangen, Germany

17 Laboratoire d'Astrophysique de Grenoble, INSU/CNRS, Université Joseph Fourier, BP 53, 38041 Grenoble Cedex 9, France e-mail: aszostek@obs.ujf-grenoble.fr

18 Institut für Astronomie und Astrophysik, Universität Tübingen, Sand 1, 72076 Tübingen, Germany

19 LPNHE, Université Pierre et Marie Curie Paris 6, Université Denis Diderot Paris 7, CNRS/IN2P3, 4 Place Jussieu, 75252 Paris Cedex 5, France

20 Charles University, Faculty of Mathematics and Physics, Institute of Particle and Nuclear Physics, V Holešovičkách 2, 180 00, Czeck Republic

21 Institut für Theoretische Physik, Lehrstuhl IV: Weltraum und Astrophysik, Ruhr-Universität Bochum, 44780 Bochum, Germany

22 University of Namibia, Private Bag 13301, Windhoek, Namibia

23 Obserwatorium Astronomiczne, Uniwersytet Jagielloński, ul. Orla 171, 30-244 Kraków, Poland

24 Nicolaus Copernicus Astronomical Center, ul. Bartycka 18, 00-716 Warsaw, Poland

25 School of Physics \& Astronomy, University of Leeds, Leeds LS2 9JT, UK

26 School of Chemistry \& Physics, University of Adelaide, Adelaide 5005, Australia

27 Toruń Centre for Astronomy, Nicolaus Copernicus University, ul. Gagarina 11, 87-100 Toruń, Poland

28 Instytut Fizyki Ja̧drowej PAN, ul. Radzikowskiego 152, 31-342 Kraków, Poland

29 Astronomical Observatory, The University of Warsaw, A1. Ujazdowskie 4, 00-478 Warsaw, Poland

30 Institut für Astro- und Teilchenphysik, Leopold-FranzensUniversität Innsbruck, 6020 Innsbruck, Austria

31 Oskar Klein Centre, Department of Physics, Stockholm University, Albanova University Center, 10691 Stockholm, Sweden

32 Oskar Klein Centre, Department of Physics, Royal Institute of Technology (KTH), Albanova, 10691 Stockholm, Sweden

33 European Associated Laboratory for Gamma-Ray Astronomy, jointly supported by CNRS and MPG 